\title{
Effects Viscous Dissipation on the Asymptotic Behaviour of Laminar Forced Convection for Herschel-Bulkley Fluid in a Circular Duct
}

\author{
Rabha Khatyr ${ }^{1}$, Jaafar Khalid-naciri ${ }^{1}$ and Ali IL Idrissi ${ }^{2}$ \\ 1. Laboratory of Mechanics, Department of Physics, Faculty of Sciences Aïn Chock, Hassan II University, B.P. 5366, Maarif, \\ Casablanca 20100, Morocco \\ 2. Laboratory of Mechanics, Department of Physics, Faculty of Sciences Ben M'Sik, Hassan II University, B.P. 7955, SidiOthmane, \\ Casablanca 20100, Morocco
}

\begin{abstract}
The asymptotic behaviour of laminar forced convection in a circular duct, for a Herschel-Bulkley fluid with constant properties, is analysed by taking into account the viscous dissipation effects. The axial heat conduction in the fluid is neglected. The asymptotic temperature field and the asymptotic value of the Nusselt number are determined for every boundary condition that allows a fully developed region. Comparisons with other existing solutions for Newtonian and non-Newtonian cases are presented.
\end{abstract}

Key words: Laminar forced convection, viscous dissipation, Herschel-Bulkley fluid, asymptotic behaviour, variable wall heat flux.

\section{Nomenclature}

$a \quad$ ratio of yield shear stress to wall shear stress

$b(R) \quad$ solution of Eqs. (19) and (20)

$\operatorname{Br}(X)$ local Brinkman number, $K u_{m}^{n+1} /\left(2 r_{0}\right)^{n} q_{\omega}(X)$

$C$ dimensionless constant employed in Eq. (18)

$c_{p} \quad$ specific heat at constant pressure

$f \quad$ function of R employed in Eq. (15)

$\mathrm{F} \quad$ function of $\mathrm{R}$ and $\mathrm{b}(\mathrm{R})$ employed in Eq. (22)

$g \quad$ arbitrary function of $\mathrm{r}$ and $x$

$K \quad$ consistency index (Pa.s)

$m \quad$ inverse of power-law exponent, $1 / n$

$n \quad$ power-law exponent

$\mathrm{Nu} \quad$ Nusseltnumber, $2 r_{0} q_{w} /\left[\lambda\left(T_{w}-T_{b}\right)\right]$

Pe Peclet number, $2 r_{0} u_{m} \rho c_{p} / \lambda$

$q_{w} \quad$ wall heat flux, $\mathrm{J} / \mathrm{s}$

$r \quad$ radial coordinate, $\mathrm{m}$

$r_{0} \quad$ radius of the tube, $\mathrm{m}$

$R$ dimensionless radial coordinate, $r / r_{0}$

$T$ temperature, $\mathrm{K}$

$T_{0} \quad$ inlet temperature distribution, $\mathrm{K}$

$u \quad$ velocity component in the axial direction, $\mathrm{m} \cdot \mathrm{s}^{-1}$

$u_{m} \quad$ mean axial velocity, $\mathrm{m} \cdot \mathrm{s}^{-1}$

Corresponding authors: Rabha Khatyr, professor, research field: fluid mechanics and heat transfer.
$U$ dimensionlessaxialvelocity, $u / u_{m}$

$x \quad$ axial coordinate, $\mathrm{m}$

$X$ dimensionless axial coordinate, $x / 2 r_{0} P e$

Greeks Symbols

$\beta \quad$ dimensionless parameter defined in Eq. (14)

$\lambda \quad$ thermal conductivity of fluid, $\mathrm{W} \cdot \mathrm{m}^{-1} \cdot \mathrm{K}^{-1}$

$\omega \quad$ dimensionless parameter, $\omega=1-2\left(\frac{a(1-a)}{m+2}+\frac{(1-a)^{2}}{m+3}\right)$

$\rho \quad$ fluid density, $\mathrm{Kg} \cdot \mathrm{m}^{-3}$

$\tau_{c} \quad$ yield shear stress, $\mathrm{Pa}$

$\tau_{w} \quad$ wall shear stress, $\mathrm{Pa}$

$\theta \quad$ dimensionless temperature, $\lambda r_{0}^{n-1}\left(T-T_{0 b}\right) / K u_{m}^{n+1}$

$\Theta \quad$ dimensionless temperature, $\left(T_{w}-T\right) /\left(T_{w}-T_{b}\right)$

Subscripts

$b \quad$ bulk quantity

$w \quad$ wall condition

$\infty \quad$ quantity evaluated for $X \rightarrow+\infty$

\section{Introduction}

Considerable attention has been devoted to convective heat transfer in non-Newtonian fluids during the past few years, mainly because of the increasing importance of these fluids in various 
chemical, processing, and nuclear industries.

Heat transfer to Herschel-Bulkley fluids in laminar flow through tubes has been investigated to some extent. Nouar et al. [1] presented a theoretical and experimental study, considering a constant wall heat flux boundary condition. In this paper, a correlation of Nusselt number is proposed taking into account the modification of the wall shear rate induced by the rheological properties, and the temperature dependent character of the fluid. In a similar study, Nouar et al. [2] obtained numerical results assuming fully developed flow at the entrance of the heated region. Two boundary conditions have been considered, constant wall heat flux and constant wall temperature. Axial conduction was neglected, and the temperature dependence of the consistency index was considered. Correlations for friction factor and Nusselt number were also proposed. Javaherdeh and Devienne [3] presented experimental and numerical results concerning heat transfer for Herschel-Bulkley fluids, the consistency of which depends on temperature. They have considered the flow through a horizontal cylindrical duct submitted to a wall cooling by an external counter current flow. They developed a simple model predicting the wall temperature distribution.

Sayed-Ahmed [4] introduced a numerical solution for laminar heat transfer of a Herschel-Bulkley fluid in the entrance region of a square duct assuming fully developed velocity profile. He solved the energy equation with dissipation effect using an implicit Crank-Nicolson method.

Analytical solutions are obtained by Pinho [5] for heat transfer in concentric annular flows of viscoelastic fluids modeled by the simplified Phan-Thien-Tanner constitutive equation. Solutions for thermal and dynamic fully developed flow are presented for both imposed constant wall heat fluxes and imposed constant wall temperatures, always taking into account viscous dissipation. Khatyr et al. [6] give analytical solutions for fully developed laminar forced convection in circular ducts for a Herschel-Bulkely fluid in a horizontal duct heated uniformly, and with various axial distributions of wall heat flux for which polynomial and logarithmic functions was considered as examples. Heat transfer with the effect of viscous dissipation for steady, laminar, both hydro-dynamically and thermally fully developed pseudo-plastic fluid through a channel of Couette-Poiseuille flow, where both the plates are kept at specified but different constant heat flux ratios being considered as thermal boundary conditions is studied by Sheela-Francisca [7]. Rashidi and Erfani [8] studied analytically the thermal-diffusion (Soret effect) and diffusion transfer of a steady MHD (magnetohydrodynamic) convective and slip flow due to a rotating disk with viscous dissipation and ohmic heating. They presented the influence of the slip parameter and the magnetic field parameter and of Eckert, Schmidt, Duforand Soret numbers on the profiles of the dimensionless velocity, temperature and concentration distributions. Rashidi et al. [9] studied analytically the effect of the buoyancy force and thermal radiation in MHD boundary layer viscoelastic fluid flow over a continuously moving stretching surface in a porousmedium. They concluded that the effect of viscoelastic parameter is to decrease the velocity and increase the temperature in boundary-layer. Abbasbandy et al. [10] presented the numerical and analytical solutions for Falkner-Skan flow of MHD Oldroyd-B fluid. They used homotopy analysis method and numerical Keller-box method. They concluded that the skin friction coefficient in Oldroyd-B fluid is larger when compared with viscous fluid, and that the relaxation and retardation times have opposite effects on the velocity components. Recently, fourth order Runge-Kutta method has been used to investigate the unsteady MHD free convective boundary-layer flow due to a permeable stretching vertical surface in a nano-fluid [11].

To our knowledge, no semi-analytical solution of the forced convection with viscous dissipation in a circular duct of Herschel-Bulkley fluid with 
no-uniform wall heat flux distribution $q_{w}(x)$ which tends to infinity for large value of $x$, is available in the literature.

The aim of the present work is to study a fully developed laminar forced convection in circular ducts for a Herschel-Bulkely fluid with viscous dissipation and negligible axial heat conduction in the fluid. The effect of the dimensionless radius of the plug core, the power-law exponent and the Brinkman number are presented and compared to those obtained in previous works.

This paper is organized as follows: in Section 2, the considered fully developed velocity profile and the energy equations are presented; Section 3 is devoted to the establishment and discussion of the results of the asymptotic behaviour of the temperature field and the Nusselt number; the conclusion is summarized in Section 4.

\section{Analysis}

Let us consider a Herschel-Bulkley fluid of constant physical properties flowing in a circular duct of radius $r_{0}$, submitted to a variable axial wall heat flux $q_{w}(x)$. The flow is supposed to be steady, laminar, fully developed and axisymetric.

The fully developed velocity profile for a laminar pipe flow of a Herschel-bulkley is given as follows [12]:

$$
u(r)=\left\{\begin{array}{c}
\frac{u_{m}}{\omega}\left(1-\left(\frac{\frac{r}{r_{0}}-a}{1-a}\right)^{m+1}\right) i f r_{c} \leq r \leq r_{0} \\
\frac{u_{m}}{\omega} \text { if } 0 \leq r \leq r_{c}
\end{array}\right.
$$

where, $\omega=1-2\left(\frac{a(1-a)}{m+2}+\frac{(1-a)^{2}}{m+3}\right), m=1 / n$ is the inverse of exponent index $n, a=\tau_{c} / \tau_{w}=r_{c} / r_{0}$ is the dimensionless radius of the plug flow region, $\tau_{c}$ the yield shear stress, $\tau_{w}$ the wall shear stress, $r$ the radial coordinate, $r_{c}$ the yield radius, and $u_{m}$ the mean value of velocity.

The energy equation and associated boundary conditions are given by Ref. [13]

$$
\begin{gathered}
\rho c_{p} u \frac{\partial T}{\partial x}=\frac{\lambda}{r} \frac{\partial}{\partial r}\left[r \frac{\partial T}{\partial r}\right]+K\left|\frac{\mathrm{d} u}{\mathrm{~d} r}\right|^{n} \frac{\mathrm{d} u}{\mathrm{~d} r}-\tau_{c} \frac{\mathrm{d} u}{\mathrm{~d} r} \\
\left.\frac{\partial T}{\partial r}\right|_{r=0}=0,\left.\quad \frac{\partial T}{\partial r}\right|_{r=r_{0}}=\frac{q_{w}(x)}{\lambda} \\
T(r, x=0)=T_{0}(r)
\end{gathered}
$$

where, $\rho, \lambda, K$ and $c_{p}$ are the density of fluid, thermal conductivity, the consistency index, and the specific heat at constant pressure, respectively.

The condition that leads to an asymptotic thermally developed region in the case of the forced convection problem described above is defined by Ref. [13]

$$
\operatorname{Lim}_{x \rightarrow+\infty} \frac{T_{w}(x)-T(r, x)}{T_{w}(x)-T_{b}(x)}=\operatorname{Lim}_{x \rightarrow+\infty} \Theta\left(r / r_{0}, x / 2 r_{0} P e\right)=\Theta_{\infty}\left(r / r_{0}\right)
$$

where, $T_{w}(x)$ and $T_{b}(x)$ are the wall temperature and the bulk temperature, respectively, $P e$ is the Peclet number, and $\Theta_{\infty}\left(r / r_{0}\right)$ is the asymptotic dimensionless temperature which is a continuous and differentiable function of $r$. The bulk value of an arbitrary function $g(r, x)$ is defined as

$$
g_{b}(x)=\frac{2}{u_{m} r_{0}} \int_{0}^{r_{0}} g(r, x) u(r) r \mathrm{~d} r
$$

If condition (5) holds, the asymptotic value of the Nusselt number $N u_{\infty}$ exists in Ref. [13] and is given by

$$
\operatorname{Lim}_{x \rightarrow+\infty} N u=2 r_{0} \operatorname{Lim}_{x \rightarrow+\infty} \frac{\left.\frac{\partial T}{\partial r}\right|_{r=r_{0}}}{T_{w}(x)-T_{b}(x)}=-\left.2 r_{0} \frac{\mathrm{d} \Theta_{\infty}}{\mathrm{d} r}\right|_{r=r_{0}}=N u_{\infty}
$$

The proof presented by Barletta [14], allows to check that the boundary value problem, expressed by Eqs. (2)-(4), has a unique solution, and that both the asymptotic behaviour of the temperature field and of the Nusselt number are independent of the inlet section temperature distribution.

Introducing the dimensionless quantities

$$
X=\frac{x}{2 r_{0} P e}, \quad R=\frac{r}{r_{0}}, \quad U(R)=\frac{u(r)}{u_{m}}, \quad \theta=\lambda r_{0}^{n-1} \frac{T-T_{0 b}}{K u_{m}^{n+1}}
$$


Eqs. (2) and (3) can be rewritten in the dimensionless form

$$
\begin{gathered}
\frac{\partial}{\partial R}\left[R \frac{\partial \theta}{\partial R}\right]=\frac{R U}{4} \frac{\partial \theta}{\partial X}+\frac{a}{(1-a)^{n+1}}\left(\frac{m+1}{\omega}\right)^{n} R \frac{\mathrm{d} U}{\mathrm{~d} R}-R\left|\frac{\mathrm{d} U}{\mathrm{~d} R}\right|^{n-1}\left(\frac{\mathrm{d} U}{\mathrm{~d} R}\right)^{2} \\
\left.\frac{\partial \theta}{\partial R}\right|_{R=0}=\left.0 \quad \frac{\partial \theta}{\partial R}\right|_{R=1}=\frac{1}{2^{n} \operatorname{Br}(X)}
\end{gathered}
$$

where, $\operatorname{Br}(X)$ is a local Brinkman number defined as:

$$
\operatorname{Br}(X)=\frac{K u_{m}^{n+1}}{\left(2 r_{0}\right)^{n} q_{\omega}(X)}
$$

Integrating Eq. (9) over the interval $0 \leq R \leq 1$ and employing Eq. (10) yields

$$
\frac{\partial \theta_{b}}{\partial X}=\frac{2^{3-n}}{B r(X)}+\frac{8}{(1-a)^{n+1}}\left(\frac{m+1}{\omega}\right)^{n}
$$

where, $\theta_{b}(X)$ is the bulk value of the dimensionless temperature $\theta(R, X)$

\section{Asymptotic Behaviour of the Temperature Field}

In this work, the asymptotic temperature field and the asymptotic Nusselt number are analyzed in the case of axial distributions of wall heat flux which yield a thermally developed region, such as

$$
\operatorname{Lim}_{X \rightarrow+\infty} \operatorname{Br}(X)=0
$$

and

$$
\operatorname{Lim}_{X \rightarrow+\infty} \frac{1}{B r(X)} \frac{\mathrm{d} B r(X)}{\mathrm{d} X}=-2 \beta
$$

where, $\beta$ is a non-vanishing positive real number.

Eq. (13) shows that the effect of viscous dissipation is negligible in the thermally developed region. Eqs. (13) and (14) are satisfied by axial wall heat flux distributions which tends to infinity when $X \rightarrow+\infty$, and which behave asymptotically as $Q(X) \mathrm{e}^{2 \beta X}, Q(X)$ can be a polynomial function, or rational function where the degree of the numerator is greater than or equal to the degree of the denominator, or any other function satisfying Eq. (13).

Therefore, in these distributions the dimensionless temperature field for large value of $X$ can be expressed by

$$
\theta(R, X)=\theta_{m}(X)+\frac{f(R)}{\operatorname{Br}(X)}
$$

By substituting Eq. (15) in Eqs. (9) and (10) and taking into account Eqs. (12)-(14), one obtains

$$
\begin{aligned}
& \frac{\mathrm{d}}{\mathrm{d} R}\left[R \frac{\mathrm{d} f}{\mathrm{~d} R}\right]=\frac{R U}{2}\left(2^{2-n}+\beta f\right) \\
& \left.\frac{\mathrm{d} f}{\mathrm{~d} R}\right|_{R=0}=\left.0 \quad \frac{\mathrm{d} f}{\mathrm{~d} R}\right|_{R=1}=\frac{1}{2^{n}}
\end{aligned}
$$

Eq. (16) can be reduced to a first-order differential equation using the following transformation

$$
f(R)=\frac{2^{2-n}}{\beta}\left[C \exp \int_{0}^{R} b\left(R^{\prime}\right) \mathrm{d} R^{\prime}-1\right]
$$

where, $C$ is a constant given by the boundary condition at $R=1$ and $b(R)$ is a continuous and differentiable function of $R$.

Substituting Eq. (18) into Eq. (16), gives

$$
R \frac{\mathrm{d} b}{\mathrm{~d} R}+b+R b^{2}=\frac{R \beta U}{2}
$$

Eqs. (17) and (18) become then

$$
b(0)=0
$$

$$
C=\frac{\beta}{4 b(1)} \exp \left(-\int_{0}^{1} b\left(R^{\prime}\right) \mathrm{d} R^{\prime}\right)
$$

Eq. (19) can also be written as:

$$
\frac{d b}{d R}=F(R, b)=-\frac{b}{R}-b^{2}+\frac{\beta U}{2}
$$

This equation with boundary condition (20) is integrated numerically using fourth-order Runge-Kutta method [15, 16]. This method is still one step, but dependent on estimates of the solution at different points, and requires 4 evaluations of function $F(R, b)$ at every time step.

$R$ is the independent variable, $b=b(R)$ is the unknown function of $R, b_{0}=b(0)$ is the given condition (Eq. (20)), and $F$ is a given 
function of $R$ and $b$ which describes the differential Eq. (22).

The variable $R$ is discretized, say $R_{i}$ for $i=0,1$, $2, \ldots$, then we determine $b_{i}=b\left(R_{i}\right)$ for $i=1,2,3, \ldots$ If $b_{i}$ is calculated, then we construct $b_{i+1}$ as follow:

$$
b_{i+1}=b_{i}+\frac{1}{6}\left(k_{1}+2 k_{2}+2 k_{3}+k_{4}\right) h
$$

with

$$
\begin{gathered}
k_{1}=h F\left(R_{i}, b_{i}\right) \\
k_{2}=h F\left(R_{i}+\frac{h}{2}, b_{i}+\frac{h k_{1}}{2}\right) \\
k_{3}=h F\left(R_{i}+\frac{h}{2}, b_{i}+\frac{h k_{2}}{2}\right) \\
k_{4}=h F\left(R_{i}+h, b_{i}+k_{3}\right)
\end{gathered}
$$

where,

$$
h=R_{i+1}-R_{i}
$$

Thereafter, the constant $C$ and the function $f(R)$ are computed by using Simpson integration method.

The asymptotic dimensionless temperature $\Theta_{\infty}(R)$ is determined by the following expression

$$
\Theta_{\infty}(R)=\frac{f(1)-f(R)}{f(1)}
$$

Taking into account Eqs. (7), (17), (18), (21) and (24), the asymptotic value of $N u$ is given by

$$
N u_{\infty}=-\left.2 \frac{\mathrm{d} \Theta_{\infty}}{\mathrm{d} R}\right|_{R=1}=\frac{2 \beta b(1)}{\beta-4 b(1)}
$$

Figs. 1a-1c represent the variation of dimensionless axial velocity $\mathrm{U}$ versus $R$ for different values of $a$ and $n$.

The obtained asymptotic values of Nusselt number are compared with those of Barletta [14] in the power-law fluid case ( $a=0$ ) (Table 1). We can note that the comparison in the power-law case is very satisfactory. Figs. 2a-2c show the asymptotic temperature profile for different values of $\beta$ and for $a$ $=0.4$. One notes that for large values of $\beta$ such as $\beta=$ 1,000 (Fig. 2c), the temperature $\Theta_{\infty}(R)$ does not vary significantly with respect to $n$. Figs. 3a-3c represent the variation of $N u_{\infty}$ versus $\beta$ for different values of $a$ and of $n$. These figures show that for $n$ fixed, $N u_{\infty}$ increases with $a$. When $\beta$ increases and $n$ decreases, the effect of the yield stress becomes important (Tables 2 and 3).

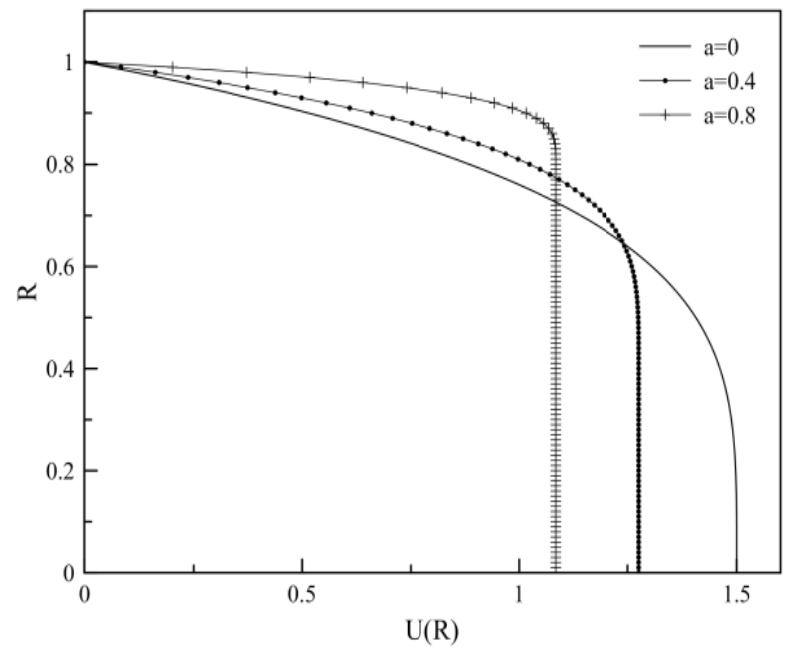

(a) $n=1 / 3$

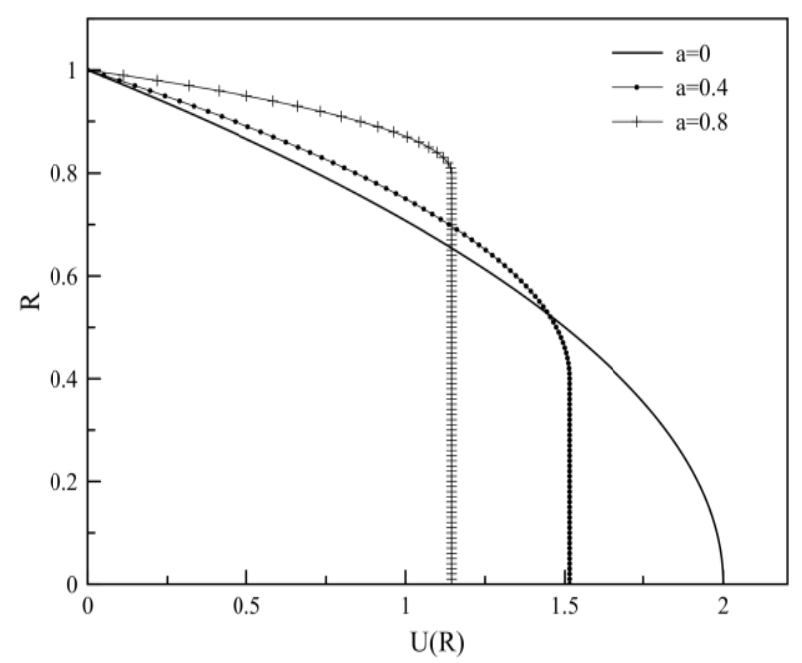

(b) $n=1$

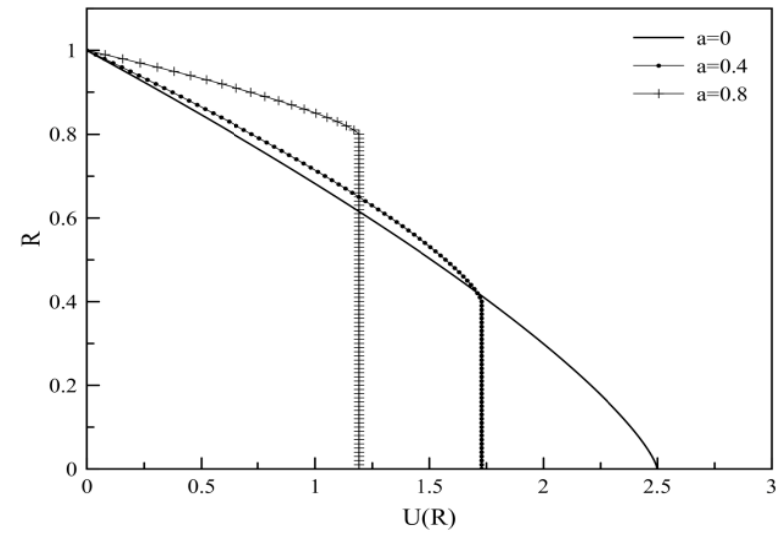

(c) $n=3$

Fig. 1 Velocity profiles used in the simulations. 
Table 1 Values of $N u_{\infty}$ for various values of $n$ compared with those of Barletta [14] in the power-law fluid case $(a=0)$.

\begin{tabular}{lllllll}
\hline \multirow{2}{*}{$\beta$} & \multicolumn{3}{c}{$n=1 / 5$} & \multicolumn{3}{c}{$n=3$} \\
\cline { 2 - 6 } & Present work & Barletta [14] & Present work & Barletta [14] & Present work & Barletta [14] \\
\hline 1 & 5.6141 & 5.6141 & 5.1431 & 5.1431 & 4.1324 & 4.1324 \\
5 & 5.9774 & 5.9774 & 5.4818 & 5.4818 & 4.4358 & 4.4358 \\
10 & 6.3858 & 6.3858 & 5.8613 & 5.8613 & 4.7715 & 4.7715 \\
20 & 7.0896 & 7.0897 & 6.5129 & 6.5129 & 5.3395 & 5.3395 \\
30 & 7.6865 & 7.6865 & 7.0634 & 7.0634 & 5.8131 & 5.8132 \\
40 & 8.2081 & 8.2082 & 7.5436 & 7.5436 & 6.2230 & 6.2230 \\
50 & 8.6740 & 8.6741 & 7.9717 & 7.9717 & 6.5860 & 6.5860 \\
60 & 9.0968 & 9.0969 & 8.3597 & 8.3597 & 6.9143 & 6.9144 \\
70 & 9.4851 & 9.4852 & 8.7159 & 8.7159 & 7.2145 & 7.2146 \\
80 & 9.8452 & 9.8453 & 9.0459 & 9.0459 & 7.4920 & 7.4921 \\
90 & 10.1817 & 10.1818 & 9.3542 & 9.3542 & 7.7508 & 7.7509 \\
100 & 10.4982 & 10.4983 & 9.6439 & 9.6439 & 7.9937 & 7.9938 \\
200 & 12.9731 & 12.9732 & 11.9067 & 11.9067 & 9.8822 & 9.8824 \\
500 & 17.4740 & 17.4740 & 16.0133 & 16.0133 & 13.2900 & 13.2902 \\
1,000 & 22.0460 & 22.0461 & 20.1794 & 20.1794 & 16.7352 & 16.7354 \\
10,000 & 48.3120 & 48.3125 & 44.0751 & 44.0751 & 36.4280 & 36.4282 \\
\hline
\end{tabular}

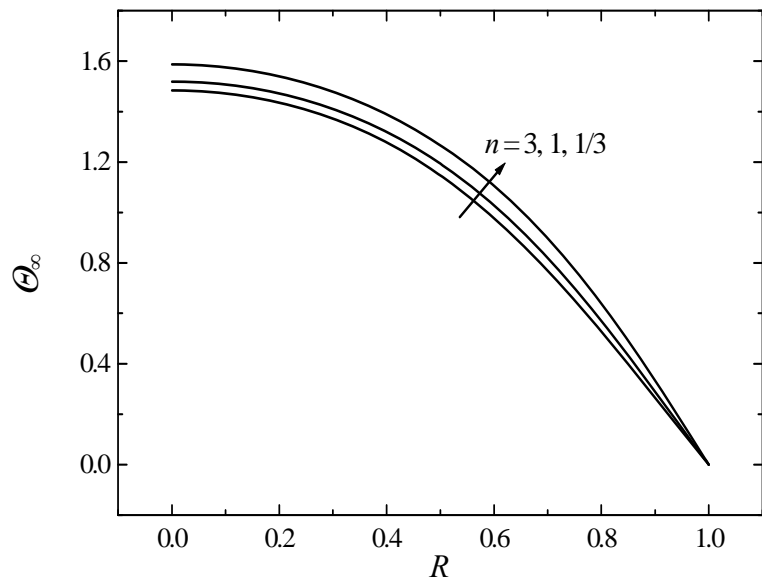

(a) $: \beta=10$

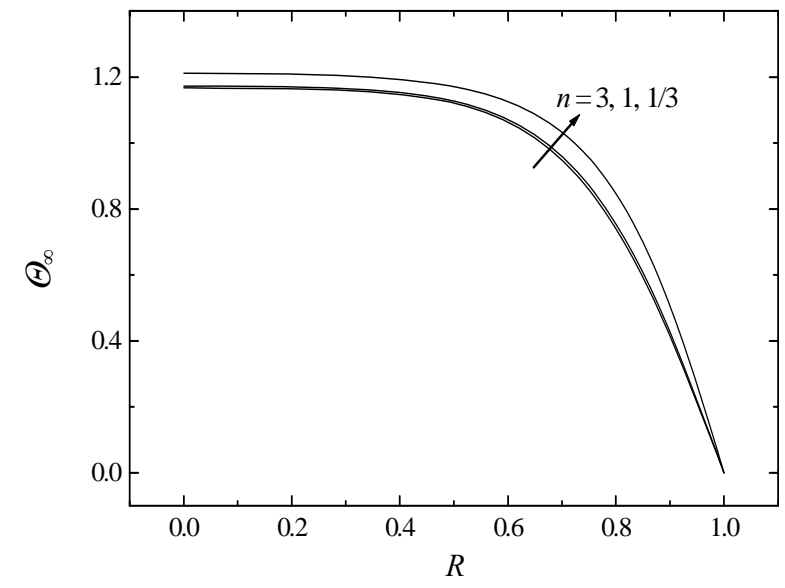

(b) : $\beta=100$

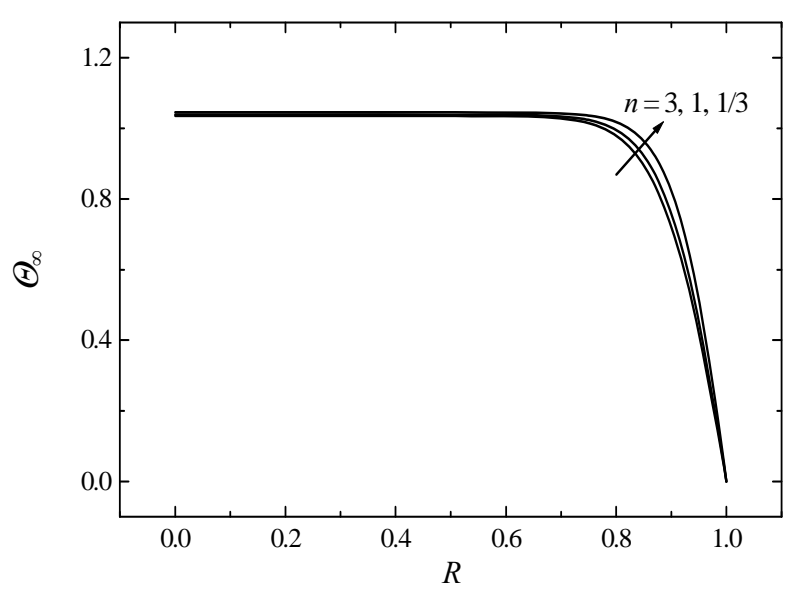

(c) $\beta=1,000$

Fig. 2 Evolution the $\Theta_{\infty}(R)$ for various values of $n$ and $\beta$, and for $a=0.4$. 


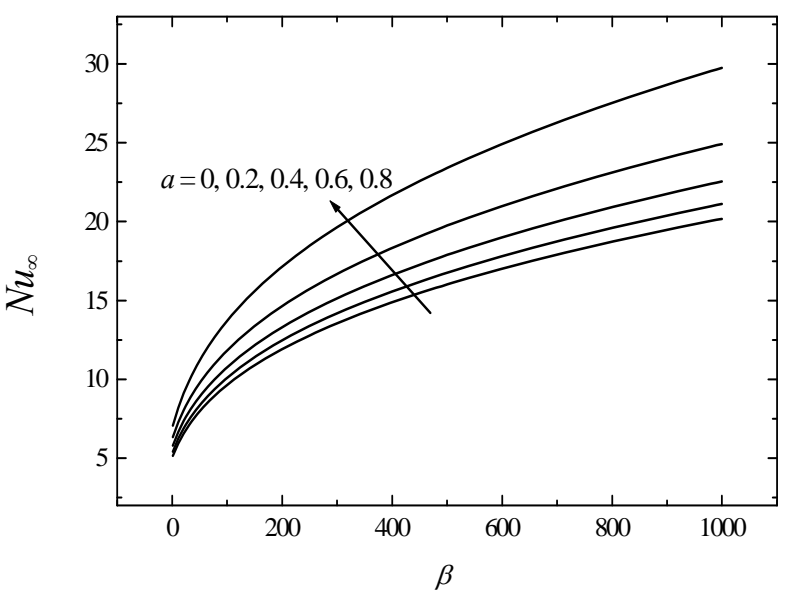

(a) $: n=1 / 3$

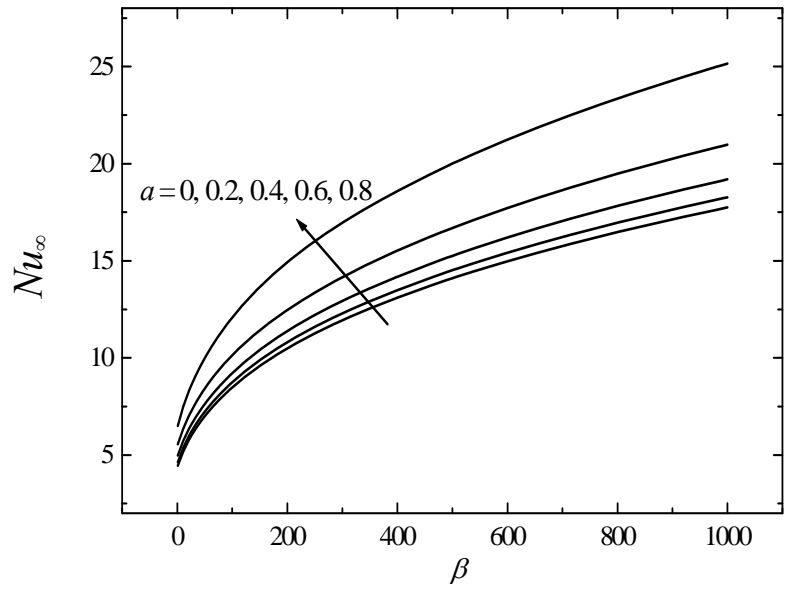

(b) $: n=1$

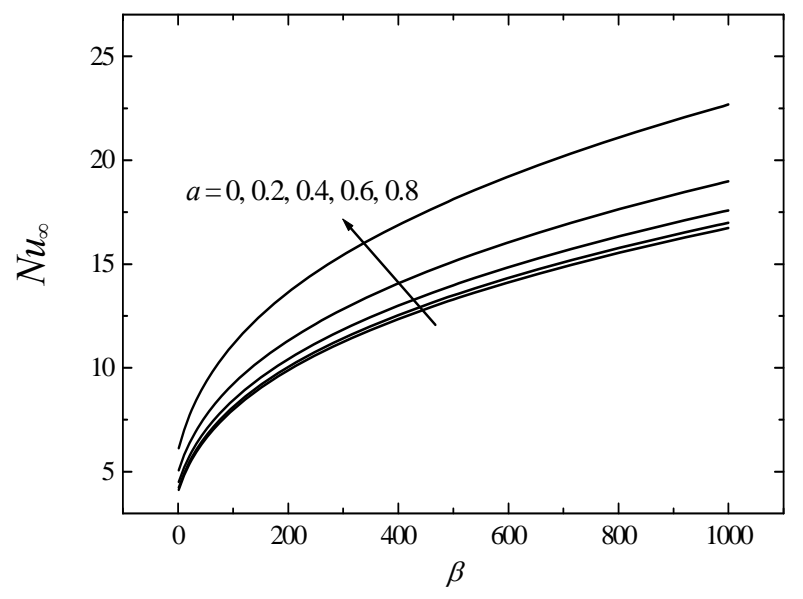

(c) $: n=3$

Fig. 3 Variation of $N u_{\infty}$ versus $\beta$ for various values of $a$ : (a) $n=1 / 3$, (b) $n=1$ and (c) $n=3$.

Table 2 Asymptotic values of $\mathrm{Nu}$ for various values of $\beta$ and $a$, and for $n=1 / 3$.

\begin{tabular}{llllll}
\hline$\beta$ & $a=0$ & $a=0.2$ & $a=0.4$ & $a=0.6$ & $a=0.8$ \\
\hline 1 & 5.1431 & 5.4157 & 5.7934 & 6.3201 & 7.0691 \\
5 & 5.4818 & 5.7652 & 6.1624 & 6.7273 & 7.5491 \\
10 & 5.8613 & 6.1576 & 6.5775 & 7.1849 & 8.0916 \\
20 & 6.5129 & 6.8333 & 7.2937 & 7.9765 & 9.0367 \\
30 & 7.0634 & 7.4054 & 7.9014 & 8.6496 & 9.8454 \\
40 & 7.5436 & 7.9051 & 8.4329 & 9.2390 & 10.5569 \\
50 & 7.9717 & 8.3511 & 8.9077 & 9.7658 & 11.1949 \\
60 & 8.3597 & 8.7557 & 9.3385 & 10.2442 & 11.7756 \\
70 & 8.7159 & 9.1272 & 9.7345 & 10.6839 & 12.3101 \\
80 & 9.0590 & 9.4717 & 10.1016 & 11.0916 & 12.8070 \\
90 & 9.3542 & 9.7935 & 10.4448 & 11.4729 & 13.2717 \\
100 & 9.6439 & 10.0960 & 10.7674 & 11.8314 & 13.7093 \\
200 & 11.9067 & 12.4610 & 13.2914 & 14.6359 & 17.1407 \\
500 & 16.0133 & 16.7582 & 17.8824 & 19.7383 & 23.3965 \\
1,000 & 20.1794 & 21.1208 & 22.5471 & 24.9243 & 29.7537 \\
10,000 & 44.7351 & 46.1638 & 49.3499 & 54.7492 & 66.2931 \\
\hline
\end{tabular}

Table 3 Asymptotic values of $\mathrm{Nu}$ for various values of $\beta$ and $a$, and for $n=3$.

\begin{tabular}{llllll}
\hline$\beta$ & $a=0$ & $a=0.2$ & $a=0.4$ & $a=0.6$ & $a=0.8$ \\
\hline 1 & 4.1324 & 4.2393 & 4.5164 & 5.0840 & 6.1377 \\
5 & 4.4358 & 4.5415 & 4.8154 & 5.3939 & 6.5156 \\
10 & 4.7715 & 4.8767 & 5.1493 & 5.7413 & 6.9385 \\
20 & 5.3395 & 5.4461 & 5.7208 & 6.3386 & 7.6655 \\
30 & 5.8331 & 5.9225 & 6.2023 & 6.8438 & 8.2795 \\
40 & 6.2230 & 6.3354 & 6.6217 & 7.2849 & 8.8143 \\
50 & 6.5863 & 6.7021 & 6.9952 & 7.6786 & 9.2904 \\
60 & 6.9143 & 7.0334 & 7.3335 & 8.0358 & 9.7210 \\
70 & 7.2145 & 7.3368 & 7.6437 & 8.3638 & 10.1158 \\
80 & 7.4920 & 7.6175 & 7.9310 & 8.6680 & 10.4809 \\
90 & 7.7508 & 7.8793 & 8.1993 & 8.9522 & 10.8216 \\
100 & 7.9937 & 8.1250 & 8.4514 & 9.2195 & 11.1413 \\
200 & 9.8822 & 10.0373 & 10.4173 & 11.3105 & 13.6280 \\
500 & 13.2900 & 13.4911 & 13.9773 & 15.1144 & 18.1187 \\
1,000 & 16.7352 & 16.9843 & 17.5825 & 18.9770 & 22.6725 \\
10,000 & 36.4280 & 36.9584 & 38.2165 & 41.1323 & 48.8644 \\
\hline
\end{tabular}




\section{Conclusions}

Laminar and hydrodynamically developed forced convection of a Herschel-Bulkley fluid flowing in a circular tube with a prescribed axial distribution of wall heat flux has been studied. The effect of viscous dissipation has been taken into account, while the axial heat conduction in the fluid has been considered as negligible. It has been supposed that, when $x \rightarrow+\infty$, $q_{w}(x)$ tends to infinity, while $\left(1 / q_{w}(x)\right)\left(\mathrm{d} q_{w}(x) / \mathrm{d} x\right)$ tends to a positive constant. If these conditions are fulfilled, the effect of viscous dissipation becomes negligible in the thermally developed region and the asymptotic value of the Nusselt number is a function of $n, a$ and the dimensionless parameter $\beta$. The asymptotic values of the Nusselt number $N u$ have been evaluated numerically for some values of $n, a$ and $\beta$. The comparisons between our theoretical results and those published in the literature for the Newtonian fluid case and the non-Newtonian fluid case (power-law fluid) show very close agreement.

\section{References}

[1] Nouar, C., Devienne, R., and Lebouché, M. 1994. "Convection Thermique pour un Fluide de Herschel-Bulkley dans la Région d'entrée d'une Conduite.” Int. J. Heat Mass Transfer 37: 1-12.

[2] Nouar, C., Lebouché, M., Devienne, R., and Riou, C. 1995. "Numerical Analysis of the Thermal Convection for Herschel-Bulkley Fluids.” Int. J. Heat Fluid Flow 16: 223-32.

[3] Javaherdeh, K., and Devienne, R. 1999. “Transfert Thermique pour L'écoulement en Canalisation Cylindrique de Fluides à Seuil: Cas du Refroidissement à Coefficient D'échange Constant.” Int. J. Heat Mass Transfer 42: 3861-71.

[4] Sayed-Ahmed, M. E. 2000. "Laminar Heat Transfer for Thermally Developing Flow of a Herschel-Bulkley Fluid in a Square Duct.” Int. Comm. in Heat Transfer 27 (7): 1013-24.
[5] Pinho, F. T., and Coelho, P. M. 2006. "Fully-Developed Heat Transfer in Annuli for Viscoelastic Fluids with Viscous Dissipation.” J. Non-Newtonian Fluid Mech. 138: 7-21.

[6] Khatyr, R., and Il Idrissi, A. 2010. “Analytic Solutions for the Forced Convection Flow of Herschel-Bulkely Fluid in a Circular Duct with Variable Wall Heat Flux.” Phys. Chem. New 55: 38-42.

[7] Sheela-Francisca, J., Tso, C. P., Hung, Y. M., and Rilling, D. 2012. "Heat Transfer on Asymmetric Thermal Viscous Dissipative Couette-Poiseuille Flow of Pseudo-Plastic Fluids.” J. Non-Newtonian Fluid Mech. 169-170: 42-53.

[8] Rashidi, M. M., and Erfani, E. 2012. “Analytical Method for Solving Steady MHD Convective and Slip Flow Due to a Rotating Disk with Viscous Dissipation and Ohmic Heating.” Engineering Computations 29 (6): 562-79.

[9] Rashidi, M. M., Momoniat, E., and Rostani, B. 2012. "Analytic Approximate Solutions for MHD Boundary-Layer Viscoelastic Fluid Flow over Continuously Moving Stretching Surface by Homotopy Analysis Method with Two Auxiliary Parameters.” Journal of Applied Mathematics 2012 (11): 853-62.

[10] Abbasbandy, S., Hayat, T., Alsaedi, A., and Rashidi, M. M. 2014. "Numerical and Analytical Solutions for Falkner-Skan Flow of MHD Oldroyd-B Fluid.” Int. J. of Numerical Methods for Heat \& Fluid Flow 24 (2): 390-401.

[11] Freidoonimehr, N., Rashidi, M. M., and Mahmud, S. 2015. "Unsteady MHD Free Convective Flow past a Permeable Stretching Vertical Surface in a Nano-fluid.” Int. J. of Thermal Sciences 87: 136-45.

[12] Skelland, A. H. P. 1967. Non-Newtonian Flow and Heat Transfer. New York: Wiley.

[13] Bejan, A. 1984. Convection Heat Transfer, 1st edition. New York: Wiley.

[14] Barletta, A. 1997. "Fully Developed Laminar Forced Convection in Circular Ducts for Power-Law Fluids with Viscous Dissipation.” Int. J. Heat Mass Transfer 40: $15-26$.

[15] Nougier, J. P. 1983. Méthodes de Calcul Numérique. Paris: Masson.

[16] Gourdin, A., and Boumahrat, M. 1989. "Méthodes Numériques Appliquées.” Technique et Documentation (Lavoisier). 